\title{
Die Europäische Union auf dem Weg aus der Krise? Eine institutionelle Nachlese zur Europawahl 2014
}

\author{
von Frank Schorkopf
}

Die zurückliegende Europawahl war mit Erwartungen aufgeladen, die Europäische Union hin auf ein parlamentarisches Regierungssystem auszurichten. In der neuen Politikperiode 2014-2019 sind Parlament, Kommission und Europäischer Rat nun auf neue Weise in ihrer politischen Ökonomie miteinander verbunden. Die Organe sind aber weiterhin herausgefordert, der Politisierung der Union mit Recht einen Ordnungsrahmen zu setzen und einer ,verhandelten Normativität" entgegen zu treten. Gegenbewegungen $z u$ erfolgreicher Integration sind keine Desintegration, sondern zeigen an, dass die europäische Integration einen Dichtegrad erreicht hat, in dem die Suche nach dem richtigen Gleichgewicht von Zentralität und Partikularität eine der größten Aufgaben ist.

The recent elections to the European Parliament were seen as a further step in moving the European Union towards a truly parliamentary political system. As regards their political economy, Parliament, Commission and Council are by now closer interwoven. The challenge ahead consists in securing a legal frame that opposes "negotiated normativity" as a result of the growing politicisation of the Union. Here, trends to oppose successful integration are not to be seen as disintegration but as a sign of a lacking balance between centralisation and particularity.

\section{Neuer Standard und veränderte Politikbedingungen}

Die Europawahl ist geschafft, die achte Legislatur des Europäischen Parlaments begonnen, die Europäische Kommission im Amt. Mit großen Erwartungen ist die neue Politikperiode 2014-2019 befrachtet. Denn die zurückliegende Europawahl steht auch für eine institutionelle Zäsur. Die Wahl war früh mit Gedanken verknüpft worden, das institutionelle Gleichgewicht in der Europäischen Union stärker in Richtung auf ein parlamentarisches Regierungssystem hin staatsanalog zu verändern. ${ }^{1}$ Der Europawahlkampf sollte Gesichter bekommen, Kandidaten,

1 Brok, E./Selmayr, M.: Der „Vertrag der Parlamente“ als Gefahr für die Demokratie?, in: integration, 31 (2008), 217-234, hier 230; zur Rolle des Generalsekretärs des Europäischen Parlaments vgl. Klaus Welle: Democratic progress, citizen's empowerment at the European level, Rede vom 17.09.2013 auf dem RAND Europe Expert Seminar „Global Societal Trends and the EU“, http://www.europarl.euro- 
die sich mit politischen Ideen um Ämter in den Brüsseler EU-Organen bewerben, durch den Wahlakt legitimiert werden und dadurch ein entsprechendes Gestaltungsmandat erhalten. Politische Entscheidungen sollten in einem politischen Modus getroffen werden. ${ }^{2}$

Als Legitimationsbrücke dient die Investitur der Europäischen Kommission. Deren Präsident kommt auf Vorschlag des Europäischen Rates, der das politische Ergebnis der Europawahl zu berücksichtigen hat, und nach Zustimmung durch das Plenum des Parlaments in sein Amt (Art. 17 Abs. 7 EUV). Beide Organe entscheiden mit Mehrheit; das notwendige Einvernehmen über den Kandidaten führt praktisch zu einer vorherigen Fühlungnahme von Europäischem Rat und Europäischem Parlament. ${ }^{3}$ Die europäischen Dachparteien können also mit jeweils ihrem Spitzenkandidaten antreten, von denen der „Wahlgewinner“ zum Kommissionspräsidenten werden soll. Das Parlament macht die Unterstützung des Kandidaten davon abhängig, welche politischen Initiativen er ergreifen wird. Der Schritt liegt nahe, dass das Parlament die Übernahme bestimmter politischer Vorhaben durch die zukünftige Kommission verlangt, ${ }^{4}$ die es aus eigener Kraft nicht durchsetzen kann.

Dass es sich bei dieser Konstruktion der europäischen Spitzenkandidatur um eine grandiose neue Erzählung handelt, zeigt sich auf den zweiten Blick. Zunächst gibt es nicht den einen Wahlgewinner. Es gibt weiterhin kein einheitliches Wahlrecht zum Europäischen Parlament. Die Unionsbürger in jedem Mitgliedstaat wählen jeweils ihr nationales Kontingent an Abgeordneten. Folglich kennen die nationalen Listen eigene Spitzenkandidaten, die im Wahlkampf auch als solche wahrgenommen werden. Der Gesamtspitzenkandidat der europäischen Dachparteien kann formal nur auf seiner nationalen Liste stehen - oder kandidiert, wie Jean-Claude Juncker, überhaupt nicht für das Europäische Parlament. Das rechnerisch aggregierte Wahlergebnis aus allen 28 Mitgliedstaaten wirft dann zwar einen Wahlgewinner aus, doch ist damit kaum etwas über den Rückhalt in den politischen Teilräumen der Europäischen Union ausgedrückt. Die

pa.eu/the-secretary-general/de, sowie Kafsack, H./Mussler, W.: Die EU spricht deutsch, in: F.A.Z., 26.06.2014.

$2 \mathrm{Zu}$ diesem Gedanken der rechtlichen Bedingungen politischen Entscheidens in der Europäischen Integration und seiner Legitimation Grimm, D.: Europa: Ja - aber welches?, in: Merkur, 68 (2014), 10451058 , hier $1054 \mathrm{ff}$.

3 Siehe die 11. Erklärung zu Artikel 17 Absätze 6 und 7 des Vertrags über die Europäische Union zum Vertrag von Lissabon, AB1. EU 2008 Nr. C 115/342.

4 Inhaltlich bieten sich die politischen Themen an, die das Parlament mit seinen Initiativberichten besetzt hat, vgl. Art. 225 AEUV und Art. 45 f. GO-EP. 
Wahlbeteiligung liegt zwischen $13 \%$ in der Slowakei und $89,6 \%$ im (wahlpflichtigen) Belgien. ${ }^{5}$ In anderen Mitgliedstaaten verweigern sich Parteien der Idee des Spitzenkandidaten, wieder andere Mitgliedstaaten sehen in der Idee die Möglichkeit, mit dem in anderen Zusammenhängen verpönten Argument der Staatsangehörigkeit „einen der ihren“ in das Präsidentenamt zu bringen. Dass die Unionsbürger den Kommissionspräsidenten mit ihrer Entscheidung unionsrechtlich nicht wählen können, sondern allenfalls eine politische Kausalkette knüpfen, fällt für einen deutschen Beobachter mit Blick auf die Praxis der Bundestagswahlkämpfe und die Kanzlerwahl dabei gar nicht entscheidend ins Gewicht.

Gleichwohl würde es der europapolitischen Logik nicht gerecht, die Idee der Spitzenkandidatur für das Amt des Kommissionspräsidenten allein nach diesen Kriterien zu beurteilen. Die europäische Integration geht häufig kleine Schritte und Umwege, verfolgt aber beharrlich ihr Ziel: Die Europawahl 2014 war Teil einer Strategie, die gegenwärtige Krise der Europäischen Union zu überwinden und Schlussfolgerungen zu ziehen aus dem gekündigten „permissiven Konsens“ der Bürger, der das europapolitische Handeln ihrer Eliten Jahrzehnte trug. ${ }^{6}$ Der Vertrag von Lissabon hat die entsprechenden Rechtsgrundlagen geschaffen, die wegen seines verspäteten Inkrafttretens, das auf die Europawahlen im Jahr 2009 erst folgte, kaum beachtet worden waren. Keine Europawahl wird mehr ohne Spitzenkandidaten auskommen. Es zeichnet sich eine Architektur für Europawahlkämpfe, zugeschnitten auf einen Gegensatz zweier Kandidaten, einschließlich medialer Rituale $a b{ }^{7}$ Die Praxis hat den EU-Vertrag ausgeschöpft und einen neuen Standard gesetzt. Die Staats- und Regierungschefs werden bei der nächsten Wahl weniger von der Bestimmtheit der Parlamentarier überrascht sein und sich frühzeitiger mit der Personalie „Kommissionspräsident“ befassen.

Während das Europäische Parlament beim institutionellen Rahmen in die Vorhand gekommen ist, ist es bei der politisch-inhaltlichen Dimension der Europawahl 2014 in die Defensive geraten. Bereits im Wahlkampf richteten etablierte Kräfte im Parlament, getragen von Wahlumfragen, die Aufmerksamkeit auf den

5 Umfangreiche Zahlen zur Europawahl 2014 sind zugänglich unter http://www.europarl.europa.eu/ elections2014-results/de/election-results-2014.html.

6 Zu diesem Gedanken stillschweigender Zustimmung Lindberg, L.N./Scheingold, S. A.: Europe's WouldBe Polity. Patterns of Change in the European Community, Englewood Cliffs, 1970, 41; Schäfer, A.: Nach dem permissiven Konsens. Zum Demokratiedefizit der Europäischen Union, in: Leviathan, 34/3 (2006), 350-376.

7 Näher zum Wahlkampf und den Wahlprogrammen Hrbek, R.: Europawahl 2014: Kontinuität und neue Facetten, in: integration, 3 (2014), 205-227, hier 208ff. 
möglichen Wahlerfolg ,populistischer“ politischer Strömungen. Nach dem Ergebnis der Europawahl steht fest, dass das Europäische Parlament nunmehr - wie etwa das Misstrauensvotum gegen die Kommission im November 2014 zeigt $^{8}$ über eine echte innerparlamentarische Opposition verfügt. Es ist eine Frage des Standpunkts und Geschmacks, diese Entwicklung politisch zu beurteilen. Das Wahlergebnis wird aber auch institutionelle Folgen haben. Denn zum einen hat sich im Parlament nun auch formal eine Große Koalition gebildet, die ihr Proprium in der politischen Bekämpfung „europafeindlicher“ Positionen sieht und insoweit mit hohen Kosten den integrationsfreundlichen Kurs halten will. Zum anderen könnte der aus dem Konstitutionalismus historisch vertraute Gegensatz von Parlament und Exekutive, von dem das institutionelle Gefüge der Europäischen Union noch geprägt ist, durch die neue Zusammensetzung aufbrechen. Denn sowohl im Parlament als auch unter den Mitgliedstaaten und damit im Ministerrat sowie im Europäischen Rat sind signifikante politische Kräfte auszumachen, die ein europapolitisches Konzept gegen den klassischen Integrationskonsens eines „Europas der Gründerväter“ vertreten.

Einstweilen ist offen, ob es bei der mehrheitlich getragenen Politik einer Eindämmung dieser politischen Kräfte als europapathologische Fälle bleibt, oder ob die neue Zusammensetzung des Europäischen Parlaments, die immerhin auch in dieser Hinsicht für den Willen der Unionsbürger steht, einen produktiven Politisierungsschub mit sich bringt. Möglicherweise werden die politischen Dachverbände bei zukünftigen Europawahlen dann stärker die inhaltliche Differenz der beiden Spitzenkandidaten betonen, ${ }^{9}$ so dass der Wähler eine politische Richtungsentscheidung treffen könnte.

Parlament, Kommission und Europäischer Rat haben die Rechnung aber möglicherweise ohne einen vierten Akteur gemacht. Nach wenigen Wochen in der neuen Wahlperiode bestimmt der Präsident der Europäischen Zentralbank, Mario Draghi, die finanz- aber auch die allgemein-politischen Schlagzeilen. Das lässt sich als eine Mandatsüberschreitung deuten, überzeugender ist momentan aber noch die These, dass die Europäische Zentralbank den der Integration eingeschriebenen, technokratischen Modus von der Kommission ein Stück weit übernommen hat.

8 Art. 234 AEUV. Der Misstrauensantrag im November 2014 war von 76 Mitgliedern der Fraktion „Europa der Freiheit und der direkten Demokratie“ (EFDD) und der Gruppe der fraktionslosen Abgeordneten gestellt worden. Das Plenum lehnte den Antrag am 27.11.2014 mit 408 gegen 101 Stimmen bei 88 Enthaltungen $\mathrm{ab}$.

9 Ausdrücklich in diese Richtung Schulz, M.: Der gefesselte Riese, Berlin, 2013, $172 \mathrm{f}$. 


\section{Konzept verhandelter Normativität}

Die Europawahl 2014 stand im Zeichen der Krise. Als die letzte BarrosoKommission im Jahr 2009 in das Amt kam, lag der Bankrott Griechenlands zwar noch gut ein Jahr voraus. Die Europäische Union war jedoch seit Herbst 2007 bereits mit den Folgen der Finanzmarktkrise beschäftigt. Die Große Krise der Europäischen Union, zu der sich die Finanzmarkt- und die Staatsschuldenkrise entwickelten, ${ }^{10}$ hat Strukturprobleme supranationaler Integration offen gelegt. Mit der personalisierten Europawahl gingen deshalb auch Erwartungen einher, der Völkervertretung eine noch größere Rolle in der Rechtfertigung europäischer politischer Herrschaft zu geben, um die für viele Menschen harten wirtschaftsund haushaltspolitischen Maßnahmen in einigen (Programm-)Mitgliedstaaten durch einen europäischen Willen entweder zu rechtfertigen oder aber diesem Mehrheitswillen entsprechend anzupassen. Das Augenmerk gilt einer Wechselwirkung zwischen der technischen Seite der Krisenbewältigung und deren politischen Kontextfolgen, die beim ersten Zug - dem Schachspiel ähnlich - bereits mitgedacht sein wollen.

Die Europäische Union ist ein rechtsstaatsfähiger politischer Raum. Sie ist mit ihrer Idee der Rechtsgemeinschaft aber auch an die Grenzen des politischen Grundkonsenses gestoßen. Die Integrationspraxis der Krise zeigt, dass EuroStaaten derzeit nicht bereit oder in der Lage sind, wirtschaftliche und soziale Reformen durchzuführen, nur weil Unionsrecht sie dazu verpflichtet. Die Union hat versucht, dieses Problem mit neuem Recht, wie mit Six Pack und Two Pack, ${ }^{11}$ mit neuen Instrumenten der Bankenunion, mit völkerrechtlichen Verträgen wie dem Fiskalpakt, aber auch mit der Aufweichung von Normativität zu lösen.

Mit besonderer Empfindsamkeit wird in Deutschland wahrgenommen, dass die Krise ein unterschiedliches kulturelles Verständnis von Recht offen legt. ${ }^{12}$ Der Selbststand des Rechts wird zwar allgemein akzeptiert, soweit Recht als handwerkliche Ressource eingesetzt wird, um politische Entscheidungen für den administrativen Vollzug zu reformulieren. Die substantielle Dimension des Rechts als eine

10 Einen glänzenden Überblick geben Peet, J./La Guardia, A.: Unhappy Union, London, 2014; aus transatlantischer Perspektive Bastasin, C.: Saving Europe, Washington, 2012, $100 \mathrm{ff}$.

11 Ioannidis, M.: EU Financial Assistance Conditionality after „Two Pack“, in: ZaöRV, 74 (2014), 61-105; Antpöhler, C.: Emergenz der europäischen Wirtschaftsregierung - Das Six Pack als Zeichen supranationaler Leistungsfähigkeit, in: ZaöRV, 72 (2012), 353-395.

12 Siehe zu dieser These bereits Schorkopf, F.: Die EU zwischen Vertragsunion und Maßnahmestaat: Ist Europa ein rechtsstaatsfähiger Raum oder Raum problembezogenen Einzelfallhandelns?, in: Nonnenmacher, G./Rödder, A. (Hg.), Wertewandel in Europa?, Frankfurt a.M., 2014, 21-24. 
der Politik maßstabsetzende und den politischen Gestaltungswillen begrenzende Ressource wird hingegen nicht in allen Mitgliedstaaten geteilt. Das Recht stößt sich an kulturellen und politischen Traditionen, an Mentalitäten. ${ }^{13}$

Es ist nicht Gegenstand dieses Beitrages, einzelne Maßnahmen und Handlungen auf ihre Rechtmäßigkeit zu prüfen. Einzelne Dossiers wie die fortlaufende Verletzung der beiden Defizitkriterien des Stabilitäts- und Wachstumspaktes sind unbestritten, bei anderen - wie den Anleihekäufen der Europäischen Zentralbank - stehen sich auf beiden Seiten wohlbegründete Standpunkte gegenüber. Auf deren autoritative Würdigung zunächst durch den Europäischen Gerichtshof im Frühjahr 2015 und sodann erneut durch das Bundesverfassungsgericht ${ }^{14}$ müssen wir warten. Die Schlussanträge des Generalanwalt Cruz Villalón vom 14. Januar 2015 deuten allerdings in die erwartete Richtung, dass die Europäische Zentralbank im Ergebnis ihre Strategie weiter verfolgen kann. Sicherlich wird ihr Handeln am Grundsatz der kompetenziellen Verhältnismäßigkeit gemessen werden, d.h. sie handelt innerhalb ihres Mandats, wenn ihre Maßnahmen verhältnismäßig sind. Darin liegt eine Rechtsbindung der Zentralbank, die in Frankfurt nicht durchweg begrüßt werden wird, und deren Bedeutung in ersten Reaktionen noch nicht erkannt worden ist. Auch wird währungspolitische Kommunikation von Zentralbankorganen als eine Rechtshandlung eingestuft, die grundsätzlich vor Gericht überprüft werden kann. Zugleich erkennt der Generalanwalt die Grenzen der Rechtsbindung an, indem er ausführt, allein die EZB verfüge über die erforderlich Erfahrung und Spezialisierung auf einem ,hochgradig technischen Terrain. "15 Die Schweizerische Nationalbank und die Devisenmärkte haben daraus ihre Schlussfolgerungen gezogen, wie auch die Europäische Zentralbank selbst. Sie beschloss am 22. Januar 2015 ihr erweitertes Programm zum Ankauf von Vermögenswerten. ${ }^{16}$ Damit sind mittelbar auch die Erwartungen ausgedrückt, was aus Karlsruhe „nun noch kommen mag“. ${ }^{17}$

13 Geppert, G.: Ein Europa, das es nicht gibt, Berlin, 2013, 67.

14 Siehe den Vorlagebeschluss des BVerfG vom 14.01.2014 im sog. EZB-/OMT-Verfahren, BVerfGE 134, 366ff.; zur Vorgeschichte Häde, U.: Die Europawährung in der Finanzkrise, in: Witzleb, N. et al. (Hg.): Festschrift für Dieter Martiny zum 70. Geburtstag, Tübingen, 2014, 891-916, hier 902ff. Zu möglichen weiteren Verläufen und der Reaktion der Märkte siehe das Jahresgutachten 2014/15 des Sachverständigenrates zur Begutachtung der gesamtwirtschaftlichen Entwicklung, Wiesbaden, 2014, $131 \mathrm{ff}$.

15 Schlussanträge des Generalanwalts Pedro Cruz Villalón vom 14.01.2015, Rs. C-62/14, Rn. 111 Gauweiler u.a.

16 Europäische Zentralbank, Pressemitteilung vom 22.01.2015: ECB announces expanded asset purchase programme, with Technical Annex.

17 Die Schlussanträge, Anm. 15, adressieren jede vom Bundesverfassungsgericht formulierte Voraussetzung für eine mögliche Nichtbeanstandung des OMT-Beschlusses, siehe BVerfGE 134, 366 (417, 
Mit etwas mehr Distanz zur Tagesaktualität interessanter ist, dass das Konzept der Rechtsgemeinschaft, also einer normativen Bindung mitgliedstaatlicher und unionaler Politik an abstrakt-generelle Regeln, mehr und mehr einem Konzept verhandelter Normativität weicht.

Der im Jahr 2011 ertüchtigte Stabilitäts- und Wachstumspakt einschließlich der makroökonomischen Überwachung und des Europäischen Semesters räumen der Kommission ein erhebliches Ermessen bei der Bewertung mitgliedstaatlicher Haushalte und ihrer Wirtschaftspolitik ein. Die Ermessensvorschriften machen es der Sache nach schwer, das Handeln der Kommission überhaupt an einem normativen Maßstab zu beurteilen. Die Stellungnahme der Kommission von Ende November 2014, den fortgesetzten Regelverstößen von Belgien, Frankreich und Italien bei ihren Haushalten vorerst keine Sanktionsschritte folgen zu lassen, ${ }^{18}$ stellen das Regelwerk nunmehr für ein breiteres Publikum sichtbar in Frage. Dem Argument, den betroffenen Mitgliedstaaten „mehr Zeit zu geben“, steht die Rechtsbindung entgegen. Sie lässt andere Mitgliedstaaten zu Recht fragen, weshalb sie sich an dem Maßstab messen lassen sollen. Die Antwort darauf kann sich nicht darin erschöpfen, im Krisenmodus größere, bereits bestehende Beurteilungs- und Ermessenspielräume zu eröffnen - denn „Krise“ ist kein technokratischer Ausnahmezustand. Ähnlich ist die Lage beim Fiskalvertrag, einem völkerrechtlichen Vertrag zur Konstitutionalisierung der Haushaltsdisziplin und zum Schuldenabbau. ${ }^{19}$ Er ist aus dem öffentlichen Diskurs verschwunden, gilt als „,politisch tot“, und seine normative Kraft für die Gesamtheit der Euro-Staaten ist gering.

Stattdessen sind Erkundungsschritte zu verzeichnen, mit denen die mögliche Änderung des Rechtsrahmens der Wirtschafts- und Währungsunion ausgetestet wird. Die geschäftsführende Direktorin des Internationalen Währungsfonds äußerte sich Anfang November 2014 zur Defizitgrenze für Staatsschulden, wonach

Rn. 100). Das erweiterte, neue Ankaufprogramm berücksichtigt die Problematik der Selektivität, der Parallelität in Bezug auf Griechenland, dessen Anleihen nicht gekauft werden würden, und des Risikos für die nationalen Haushalte.

18 Commission Opinion vom 28.11.2014 on the Draft Budgetary Plan of Belgium, C (2014) 8800 final; of France C (2014) 8805; of Italy C (2014) 8806. Eine Übersicht aller Dokumente zum Europäischen Semester 2015 findet sich unter http://ec.europa.eu/economy_finance/economic_governance/sgp/ budgetary_plans/index_de.htm. Die Entscheidung liegt beim ECOFIN-Rat, siehe den Hinweis von Schäuble, $W$. Öffentliche Debatten bringen nichts, Interview mit dem Deutschlandfunk vom 28.11.2014, http://www.bundesfinanzministerium.de/Content/DE/Interviews/2014/2014-11-28-deutschlandfunk.html?view=renderPrint

19 Näher dazu Schorkopf, F.: Europas politische Verfasstheit im Lichte des Fiskalvertrages, in: ZSE, 1 (2012), 1-29. 
das $60 \%$-Kriterium überdacht und rückgesetzt werden könnte. ${ }^{20}$ Mit anderen Worten, die nach dem Stabilitäts- und Wachstumspakt erlaubte Staatsschuldenquote könnte auf 80 oder $90 \%$ des BIP heraufgesetzt werden, um das fiskalische Handeln eines Teils der Euro-Staaten wieder in Einklang mit dem Recht zu bringen und anderen eine realistische Aussicht auf zukünftige Einhaltung zu geben.

Ein zweites Beispiel ist das geldpolitische Handeln der Europäischen Zentralbank. Die Zentralbank befindet sich im dritten Zyklus ihrer Bemühungen seit dem Jahr 2010, mit geldpolitischen Mitteln die Staatsschuldenkrise zu bewältigen. Dem ersten Schritt eines Bündels von Maßnahmen zur Verbesserung der Kreditvergabe 2010-2011 folgten 2012 die Beschlüsse über langfristige, d.h. dreijährige Refinanzierungsgeschäfte sowie die Beschlüsse zu Staatsanleihekäufen über das auch praktizierte Securities Market Programme (SMP) und das nicht aktivierte - Outright Monetary Transactions (OMT) Konzept. Der dritte Zyklus ist gekennzeichnet durch eine vorbeugende geldpolitische Lockerung, mögliche umfassende Wertpapierankäufe und die nochmalige und langfristig zugesagte Senkung des Leitzinsens einschließlich von Negativzinsen. ${ }^{21}$ Doch schon hat eine Debatte begonnen, welche Maßnahmen der Zentralbank noch bleiben, wenn die geldpolitischen Ziele - Abwendung einer Deflation und Annäherung an das offizielle Inflationsziel des Eurosystems - dennoch nicht erreicht werden. Einstweilen sind Forderungen, das Verbot der unmittelbaren monetären Haushaltsfinanzierung (Art. 123 AEUV) aufzuheben, nur versprengte Einzelmeinungen. Nach einhelliger Überzeugung wäre dazu eine Vertragsänderung notwendig. Aus der ökonomischen Ideenwelt wird die Dystopie einer Abschaffung des Bargeldes ins Gespräch gebracht, u.a. mit dem technokratischen Argument, dass dann nicht nur volkswirtschaftliche Kosten gespart, sondern auch die geldpolitische Steuerung effektiver ausgestaltet werden könnte. ${ }^{22}$ Wer bislang meint, dass die Europäische Zentralbank ihr vertragliches Mandat mit den im Umfang begrenzten Staatsanleihekäufen überschritten hat, wird in Anbetracht der mit den Maßnahmen des dritten Zyklus verbundenen Risiken wohl anneh-

20 Vgl. die bei Reuters zitierte Äußerung von Christine Lagarde vom 07.11.2014 auf einer Konferenz der Französischen Nationalbank: „It could well be that some of those numbers - the $60 \%$ in particular may need some rethink and reset, with a bit of an update, considering how the situation has evolved and how the long-term debt of sovereigns has grown over time."

21 Zusammenfassende Bewertung dieser Maßnahmen im Jahresgutachten 2014/15 des Sachverständigenrates zur Begutachtung der gesamtwirtschaftlichen Entwicklung, Wiesbaden, 2014, 136-164.

22 Rogoff, K.: Costs and Benefits to Phasing Out Paper Currency, NBER Working Paper No. 20126, Mai 2014; vgl. auch: Leaving dead presidents in peace. Abolishing notes and coins would bring huge economic benefits, in: The Economist, 20.09.2014. 
men, die Zentralbank bewege sich in einem Unionsrechtsvakuum. Derweil erlauben die Bedingungen des neuen, erweiterten Ankaufprogramms der Europäischen Zentralbank vom 22. Januar 2015, so ein kolportierte These, dass EuroStaaten ihre von der nationalen Zentralbank gehaltenen Schulden durch Gesetz eines Tages restrukturieren könnten.

Diese Entwicklungen sind nicht frei von Verzweiflung der sie tragenden Akteure. Positiv gewendet ließe sich betonen, dass diese Entwicklungen aus der vergangenen Legislatur 2009-2014 auch für eine stärkere Politisierung der europäischen Integration in der angelaufenen Wahlperiode stehen. Eine solche Politisierung, die durchaus als erwünscht gesehen wird, um den technokratischen Charakter der Europäischen Union zurückzudrängen und größere Bürgernähe zu erreichen, führt allerdings auch einen noch nicht in unser Bewusstsein getretenen Strukturwandel herbei.

Eine Politisierung der Wirtschafts- und Währungsunion wird die Aufmerksamkeit der Institutionen weg von den Strukturen einer europäischen sozialen Marktwirtschaft hin zu Wohlstandsverteilung nicht nur von Nord nach Süd, sondern zugleich von unten nach oben in der Gesellschaft lenken. ${ }^{23}$ Die Verfassung der Rechtsgemeinschaft Europäische Union ist kein neutrales Organisationsstatut, sondern eine Ordnung mit einer auf Güterallokation ausgerichteten Wirtschaft zum Wohlstandserwerb im Binnenmarkt. Die gegenwärtige Debatte zwischen den Mitgliedstaaten, die sich im Europäischen Rat spiegelt, zeigt hingegen, dass es zunehmend um die Verteilung erworbenen Wohlstands zwischen den Mitgliedstaaten und ihren Gesellschaften geht. ${ }^{24}$ Dass es keinen tatsächlichen Konsens über die negativen Folgen übermäßiger Staatsverschuldung gibt, zeigt die Stellungnahme der Kommission zur deutschen Haushaltsplanung 2015. Trotz der deutlich über $60 \%$ liegenden Staatsschuldenquote wird eine zusätzliche Schuldenaufnahme für Investitionen empfohlen und u.a. mit den sehr niedrigen

23 Das die EZB durchaus Problembewusstsein hat, zeigt die Rede des EZB-Direktoriumsmitglieds Yves Mersch, Keynote, Corporate Credit Conference vom 17.10.2014, zugänglich unter http://www.ecb.europa.eu/press/key/date/2014/html/-sp141017_1.en.html.

24 Der Begriff der Solidarität spielt dabei eine zentrale Rolle, siehe die Beiträge in Calliess, C. (Hg.): Europäische Solidarität und nationale Identität, Tübingen, 2013, besonders die Beiträge von Bieber, R.: Gegenseitige Verantwortung - Grundlage des Verfassungsprinzips der Solidarität in der Europäischen Union, 67-82, und Enderlein, H.: Solidarität in der Europäischen Union - Die ökonomische Perspektive, 83-98. Zu den Finanztransfers zwischen den Mitgliedstaaten Blanke, H.-J./Pilz, S.: Solidarische Finanzhilfen als Lackmustest föderaler Balance in der Europäischen Union, in: Europarecht, 5 (2014), $541-566$. 
Zinssätzen begründet, die ,eine die Finanzierungskosten weit übertreffende soziale Rendite“" implizierten. ${ }^{25}$

Die Schlussfolgerung aus diesen Überlegungen könnte sein, anzuerkennen, dass die „Integration durch Recht“ Krisen verursachen kann. In den Mitgliedstaaten wird erkannt, dass die Europarechtstreue die innere politische Stabilität unterhöhlen würde. Nicht jeder Mitgliedstaat hat eine politische Ökonomie, die eine Agenda-Reform aushält. ${ }^{26}$ Mit anderen Worten, in ihrem Bestreben, mit Recht zu stabilisieren, destabilisiert die Europäische Union. Zugleich wird die Rechtsgemeinschaft und Wirtschaftsverfassung der Stabilität und Ordnung als unverzichtbare Voraussetzung für gelingendes Europa gesehen. Altkanzler Helmut Kohl als personifizierter Typus des klassischen „Europas der Gründerväter“ hat in seinem Essay vom Herbst 2014 mehrfach formuliert, dass es auf dem Weg zu einem geeinten Europa zu den vordringlichsten Aufgaben der Europäischen Union gehöre, im Euroraum ,schnellstmöglich wieder zu einer Gemeinschaft der Stabilität und der Rechtstreue“ zurückzukehren. ${ }^{27}$ Die europäische Integration hat sich in ein Dilemma hinein entwickelt: Ihre Dynamik benötigt politisches Ermessen, ein fallbezogenes Aushandeln von Entscheidungen. Ihr normatives Selbstbild und ihr Anspruch, verfasster politischer Raum zu sein, fordern die Rechtsunion.

\section{III. „Desintegration“ als Folge erfolgreicher Integration?}

Die Finanz- und Staatsschuldenkrise legt Bruchstellen offen und markiert den prekären Stand der kulturellen, politischen und wirtschaftlichen Integration in Europa. Die Mitglieder des Europäischen Rates sind sich nicht einig darüber, wie die Wirtschafts- und Währungsunion zu vollenden ist. ${ }^{28}$ Deutschland hat zu dieser Krise der Europäischen Union nicht nur dadurch beigetragen, dass es bei den Beitritten zur Währungsunion nicht so genau hinsah ${ }^{29}$ und 2003 die Regeln des Stabilitäts- und Wachstumspaktes als Negativvorbild - Hand in Hand mit Frank-

25 Stellungnahme der Kommission vom 28.11.2014 zur Übersicht über die Haushaltsplanung Deutschlands, C (2014) 8801, Ziff. 14.

$26 \mathrm{Zu}$ den politischen Rahmenbedingungen einer schuldenaversen, aus Steuern und Abgaben finanzierten Haushaltspolitik Armingeon, K./Guthmann, K./Weisstanner, D.: Politische Voraussetzungen von Austeritätspolitik, in: ZSE, 2-3 (2014), 242-271, mit dem für die These einer ,verhandelten Normativität“ bemerkenswerten Ergebnis, dass Verhandlungen und Kompromissbildung hocheffiziente Verfahren in modernen Gesellschaften zur Durchsetzung umfassender Politikveränderungen seien.

$27 \mathrm{Kohl}$, H.: Aus Sorge um Europa. Ein Appell, München, 2014, 78, 80, 84, 110 (Zitat).

28 Europäischer Rat, Schlussfolgerungen vom 27./28.06.2013, EUCO 104/2/13 REV 2, Ziff. 4-16.

29 Loth, W.: Kreativ, vor allem in Buchführung. Wie Athen in die Eurozone gelangte und Berlin seinen Widerstand aufgab, in: F.A.S., 18.09.2011. 
reich - missachtete. ${ }^{30}$ Seine gesellschaftlichen und politischen Eliten haben aber auch zu lange Zeit an idealisierten Integrationsleitbildern festgehalten, die zahlreiche Integrationsschritte negativ beeinflusst haben. Es ist ein Irrtum zu meinen, dass die Wirtschafts- und Währungsunion sich in stabilen und rechtstreuen Bahnen bewegen würde, wenn die beiden zitierten Fehler von einer bestimmten Bundesregierung nicht gemacht worden wären. ${ }^{31}$ Die Architektur des Euro hat wirtschaftliche Zusammenhänge geschaffen, aus denen die Euro-Staaten nicht die notwendigen Schlussfolgerungen für ihre Gesellschaften ziehen wollen, und sie nährt Erwartungen relevanter ökonomischer Kräfte, denen sich die Mitgliedstaaten nicht entziehen können. Wer den politischen Schritt zur gemeinsamen Währung dennoch wagte, der unternahm ein Völkerexperiment mit ungewissem Ausgang für Frieden und Freiheit in Europa.

Mit der bereits erwähnten Aufkündigung des „,permissiven Konsenses“ in der europäischen Bürgerschaft entsteht ein neuer Rahmen für die europäische Integration. Dieser Rahmen wird gebildet durch die Bildung und die Wahlerfolge von politischen Parteien in verschiedenen Mitgliedstaaten, die den Institutionen der Europäischen Union offen kritisch gegenüber stehen, und damit einhergehend eine europakritische öffentliche Meinung. Er wird markiert durch die ökonomische Divergenz der Mitgliedstaaten, durch deren teilweise Überforderung mit komplexen Verwaltungs- und Vollzugsstrukturen, durch die bedrohliche Aussicht, für formale Vertragsänderungen in 28 nationale Ratifikationsverfahren nebst Plebisziten gehen zu müssen, durch Griechenlands ungeklärten Status im Eurosystem, sowie durch die partikularen Standpunkte zur Supranationalität und zu bestimmten Politiken, besonders in Großbritannien, aber auch in anderen Mitgliedstaaten.

Diese Entwicklungen werden als Desintegration wahrgenommen, also als das Gegenteil der Integration, einer Einheitsbildung, die - wenn auch nicht linear, so doch in stetiger Bewegung - auf eine als positiv definierte Ganzheit zuläuft. ${ }^{32}$ Ein Integrationskonzept, das an einer ,immer engeren Union der Völker Europas" ausgerichtet ist (Art. 1 Abs. 2 EUV) interpretiert die genannten Entwicklungen als tatsächliche oder zumindest potentielle Rückschritte auf dem Fort-

30 EuGH, Rs. C-27/04, Slg. 2004, I-6649 - Kommission/Rat und das Interview mit G. Schröder, in: Passauer Neueste Nachrichten, 09.09.2011.

31 So aber die Argumentation von Kohl., H., a.a.O., 82f., wonach die Bundesregierung unter Rot-Grüner Führung die Verantwortung trage.

32 Einen Versuch theoretischer Erfassung von „Desintegration“ unter dem dialektischen Gedanken von Zug- und Gegenkräften unternehmen Eppler, A./Scheller, H. (Hg.): Zur Konzeptionalisierung europäischer Desintegration, Baden-Baden, 2013. 
schrittspfad. Die Europawahl 2014 und die vereinzelten Forderungen, die Willensbildung in der Europäischen Union zu politisieren und damit zu demokratisieren, sind auch bereits Antworten auf die Desintegrationsprozesse. Aber, handelt es sich in jedem Fall um Desintegration?

Eine freundlichere Lesart interpretierte die Entwicklungen als geradezu notwendige Folge europäischer Integration. Sie stehen für den gegenwärtigen Status der Integration und zeigen an, wie nah die Europäische Union mittlerweile an den Kern der Mitgliedstaaten als politische Primärräume herangerückt ist. ${ }^{33}$ Die Mitgliedstaaten werden gedrängt, grundlegende wirtschaftspolitische Entscheidungen zu treffen, die Folgewirkungen nach innen haben; ein Ausweichen, das noch vor der Dritten Stufe der Wirtschafts- und Währungsunion möglich war, ist wegen der Logik der Gemeinsamen Währung nicht mehr leicht möglich. Gleiches gilt für die Freizügigkeit im Binnenmarkt und die Einwanderung in die Europäische Union, deren von der Politik abstrakt gewollte Dynamik nachführende Entscheidungen der Mitgliedstaaten erfordern.

Die Europäische Union treffen diese vermeintlich desintegrativen Entwicklungen nicht unvorbereitet, wie etwa die seit dem Vertag von Lissabon bestehende Rechtsgrundlage für die Rückübertragung von Zuständigkeiten (Art. 48 Abs. 2 EUV) zeigt. Sie ist, bei verständiger Würdigung, kein verhandlungsbedingter Eintrag auf der Verlustliste europäischer Integration. Sie ist Ausdruck der Differenzierung und einer möglichen ständigen Neujustierung der Kompetenzordnung der Union. 60 Jahre nach dem Beginn der europäischen Integration mit der Gründung der Montanunion haben weder die europäische Integration noch das Europarecht einen prekären Status. Die abweisenden Reflexe gegen Argumente und Haltungen, die nicht dem herrschenden Leitgedanken entsprechen, beruhen auf der eindimensionalen Vorstellung von Integration als linearem Prozess. Die europäische Integration hat hingegen einen Dichtegrad erreicht, in dem die Suche nach dem richtigen Gleichgewicht von Zentralität und Partikularität eine der größten Aufgaben ist. ${ }^{34}$ Wenn die Gegenwartsprobleme der Europäischen Union nicht allein als Ausdruck mangelnden Integrationswillens, sondern zumindest auch als Ausdruck der Steuerungskrise sozialtechnischen Denkens gesehen wer-

33 Vgl. Kohlhase, N.: Die Europäische Gemeinschaft vor der Gefahr der Desintegration, in: Europa-Archiv 1969/I, 263-268, hier 268.

34 Die Diskussion über das Verhältnis von nationalem Recht und Europarecht sowie über Verfassungsidentität lässt sich als Ausdruck einer solchen Gleichgewichtssuche auffassen; dass dieses Anliegen nicht nur in Deutschland, sondern in zahlreichen Mitgliedstaaten verfolgt wird, zeigt eindrucksvoll Wendel, M.: Permeabilität im europäischen Verfassungsrecht, Tübingen, 2011. 
den könnten, kämen für die Politik neue Weichenstellungen in den Blick. Die Europäische Union bewegt sich dann nicht „gerade so" im erwarteten Korridor effektiver europäischer Integration, sondern sie operiert „bereits“ im Grenzbereich wirtschafts-, finanz- und sozialpolitischer Aufgaben eines primären Politikraums, mit dem die Bürger ihr Gemeinwohl organisieren und von dem sie Problemlösungen erwarten.

Mit mehr zeitlichem Abstand zur Gegenwartskrise werden wir deshalb sehen, dass beispielsweise die Bankenunion ein erheblicher Integrationsschritt ist, der dem trotzigen Leitspruch „Krise als Chance“ zur Ehre gereicht. Die Vergemeinschaftung der Bankenaufsicht, der Bankenabwicklung und der Einlagensicherung sind Schritte, die in der Normallage politisch nicht mehrheitsfähig waren. Die Bankenunion schließt über ihren Abwicklungsfonds sogar die Umverteilung von Wohlstandsressourcen mit ein. ${ }^{35}$ Die wesentlichen Sekundärrechtsakte sind mit Zustimmung des Europäischen Parlaments erlassen worden. Ob die Zuständigkeitsverteilung mit der gewählten Aufsichtskonstruktion für die Europäische Zentralbank glücklich ist, muss sich erst noch zeigen. Zudem bleibt unsicher, ob die beaufsichtigten Banken solvent sind und möglichen Belastungen tatsächlich standhalten. Jedenfalls steht das Beispiel der Bankenunion bereits für den Einfluss eines europäischen Parlamentarismus zum Ende der letzten Legislatur. In der neuen Wahlperiode 2014-2019 sollten Politiker, Funktionäre und Vordenker der europäischen Integration keine Furcht vor Desintegration haben. Sie sollten Responsivität zeigen und nach den gesellschaftlichen Funktionsbedingungen einer Integration in der Gegenwart fragen.

35 Bundesbank: Die neuen europäischen Regeln zur Sanierung und Abwicklung von Kreditinstituten, Monatsbericht, 6 (2014), 31-58, hier 42ff. 